\title{
COMPARACIÓN DE LA FUERZA ADHESIVA Y EL TIPO DE FALLA DE DOS SISTEMAS ADHESIVOS PARA ORTODONCIA
}

\author{
${ }^{1}$ Ana Lucía Ciceri, ${ }^{2}$ John Jairo Monroy Parada,${ }^{3}$ Gerardo Ardila Duarte, \\ ${ }^{4}$ Antonio Luna Salguero, ${ }^{5}$ Jaime Rodrigo Rivera Barrero. \\ ${ }^{1}$ Odontóloga U. CES, Especialista en Ortodoncia Fundación CIEO U. Militar Nueva Granada, Bogotá (Colombia). \\ ${ }^{2}$ Odontólogo U. Nacional, Estudiante III año Especialización en Ortodoncia Fundación CIEO U. Militar Nueva Granada, Bogotá (Colombia). \\ ${ }^{3}$ Licenciado en Matemáticas U. Pedagógica Nacional, Especialista en Análisis de Datos U. de la Salle, Maestría en Estadística U. Nacional, \\ Profesor Asociado Fundación CIEO U. Militar Nueva Granada, Bogotá (Colombia). \\ ${ }^{4}$ Odontólogo U. Javeriana, Especialista en Ortodoncia Fundación CIEO U. Militar Nueva Granada, Profesor asistente Fundación CIEO U. Militar Nueva Granada, Bogotá (Colombia). \\ ${ }^{5}$ Odontólogo U. Javeriana, Maestría en Biomateriales U. de Alabama, Profesor asistente Fundación CIEO U. Militar Nueva Granada, Bogotá (Colombia).
}

Autor responsable de correspondencia: Jaime Rodrigo Rivera $B$

Correo electrónico: jr.rivera@cieo.edu.co

Investigación presentada en el 89th General Session \& Exhibition of The International Association for Dental Research, San Diego, 2011

RESUMEN

Objetivo: Comparar la fuerza adhesiva y el tipo de falla de dos sistemas adhesivos para ortodoncia.

Materiales y métodos: Veinte primeros premolares superiores fueron divididos aleatoriamente en dos grupos: 10 para el grupo de grabado total (Transbond ${ }^{\mathrm{TM}}$ XT, 3M Unitek, St. Paul, Minnesota, USA) y 10 para el grupo autograbador (Transbond ${ }^{\mathrm{TM}}$ Plus Self Etching Primer + Transbond $^{\mathrm{TM}}$ XT, 3M Unitek, St. Paul, Minnesota, USA). A estos dientes se les cementaron brackets estándar (Ortho Organizers, California, USA) y después de 24 horas fueron sometidos a 5000 ciclos térmicos. Posteriormente se aplicó una fuerza de corte (velocidad $1.5 \mathrm{~mm} / \mathrm{min}$ ) en la interfase bracket-esmalte en una máquina universal de ensayos (Instron ${ }^{\mathrm{TM}}$ ). Finalmente, las superficies del esmalte y las bases de los brackets se observaron mediante un estéreo-microscopio (Stemi 2000C, Carl Zeiss, Göttingen, Germany).

Resultados: En fuerza adhesiva se observo una diferencia estadísticamente significativa $(\mathrm{P}<0.05)$ entre los dos sistemas, presentó mayor promedio el adhesivo de grabado total (28,98MPa vs. $18,67 \mathrm{MPa})$. El tipo de falla predominante fue en la interfase adhesivo-esmalte (80\% y $70 \%$ respectivamente).

Conclusión: La fuerza adhesiva y el tipo de falla están influenciados por el pre-tratamiento del esmalte y la composición química del adhesivo. La fuerza adhesiva obtenida al utilizar un sistema autograbador en ortodoncia produce suficiente retención micro-mecánica con menor riesgo de lesionar el tejido dental. [Ciceri AL, Monroy JJ, Ardila G, Luna A, Rivera JR. Comparación de la fuerza adhesiva y el tipo de falla de dos sistemas adhesivos para ortodoncia. Ustasalud 2011; 10: 29 - 35]

Palabras clave: Grabado dental, Materiales dentales, Brackets ortodónticos.

\section{BOND STRENGTH AND FAILURE MODE COMPARISON BETWEEN TWO ADHESIVE SYSTEMS FOR ORTHODONTICS}

\begin{abstract}
Objective: To compare the bond strength and failure mode between total and self-etching systems used for brackets cementation.

Methods: Twenty human first premolars were randomly divided into two groups: 10 for the total etching system (Transbond ${ }^{\mathrm{TM}}$ XT, $3 \mathrm{M}$ Unitek, St. Paul, Minnesota, USA) and 10 for the self-etching system (Transbond ${ }^{\mathrm{TM}}$ Plus Self Etching Primer + Transbond ${ }^{\mathrm{TM}}$ XT, 3M Unitek, St. Paul, Minnesota, USA). Standard brackets (Ortho Organizers, California, USA) were cemented, and after 24 hours submitted to 5000 thermal cycles. Subsequently, shear bond strength tests were performed applying force at the bracket/enamel interphase using an universal testing machine (Instron ${ }^{\circledR}$ ) at $1.5 \mathrm{~mm} / \mathrm{min}$ crosshead speed. Finally, enamel surfaces and bracket bases were observed using a stereomicroscope (Stemi 2000C, Carl Zeiss, Göttingen, Germany).

Results: The results, in terms of bond strength, showed a statistical significant difference $(\mathrm{p}<0.05)$ between the two adhesive systems, presenting higher values the total-etching system (28,98MPa vs. 18,67MPa). The predominant failure modes presented for both systems were at the adhesive-enamel interphase (80\% and $70 \%)$.

Conclusion: Bond strength and failure mode are influenced by the dental substrate pre-treatment and the chemical composition of the adhesive. Therefore, the in-vitro bond strength obtained when a self-etching system is used in orthodontics, produce enough micromechanical retention with lower risk of damage to the dental tissue.
\end{abstract}

Key words: Dental etching, Dental materials, Orthodontic brackets.

Recibido para publicación: 15 de junio de 2011. Aceptado para publicación: 29 de junio de 2011. 


\section{INTRODUCCIÓN}

La adhesión en esmalte ha sido un procedimiento clínico exitoso desde que Buonocore propuso la utilización del grabado ácido por primera vez en 1955. ${ }^{1}$ A partir de esta fecha, las diferentes áreas de la odontología se han beneficiado de este descubrimiento gracias a la optimización de los diferentes protocolos adhesivos. La ortodoncia utiliza estos protocolos con la finalidad de fijar la aparatología que permite corregir la posición de los dientes y los maxilares en busca de una armonía funcional y estética.

Los sistemas adhesivos generalmente utilizados en ortodoncia se denominan sistemas de grabado total o de quinta generación. No obstante, gracias a la evolución de los adhesivos dentales, actualmente se encuentran disponibles en el mercado, materiales que presentan propiedades físico-mecánicas que potencialmente pueden disminuir inconvenientes relacionados con la adhesión y, a su vez, reducir el tiempo de trabajo durante el procedimiento de cementación. Estos materiales, por su mecanismo de acción se conocen como autograbadores y corresponden respectivamente a la sexta y séptima generación de acuerdo a su desarrollo histórico. ${ }^{2}$ Una de las características más importantes de estos materiales es que poseen un componente hidrófilo que favorece la adhesión aún en presencia de humedad. Los sistemas adhesivos de séptima generación además de no requerir un grabado ácido previo, se encuentran disponibles en una presentación de un solo frasco o blíster.

Durante las últimas dos décadas, diferentes estudios se han concentrado en la modificación de los protocolos de adhesión convencionales, como por ejemplo: airear o no el adhesivo, utilizar diferentes concentraciones de ácido, pre-tratar con hipoclorito el esmalte o combinar los adhesivos con diferentes materiales cementantes. ${ }^{3-7}$ Así mismo, a pesar de que autores como Kaaden y colaboradores concluyeron que la fuerza adhesiva de los sistemas autograbadores en el esmalte era significativamente menor que en la dentina, ${ }^{8}$ la adhesión al tejido adamantino producida por este tipo de materiales ha sido variable principal de estudio en diversas investigaciones.

Gran parte de los reportes previos han establecido que existen diferencias estadísticamente significativas al comparar sistemas de grabado total con autograbadores, estos últimos presentan menores valores de adhesión. ${ }^{9-14}$ Sin embargo, Shono, Arnold y Dorminey y colaboradores han reportado que esta diferencia es no significativa. ${ }^{15-17}$ Esta situación ha generado gran controversia con respecto a la efectividad clínica que puedan presentar los adhesivos autograbadores de séptima generación, ya que éstos al tener mezclados en un solo frasco dos diferentes componentes: primer y adhesivo, de cierta forma pueden ver comprometido su desempeño en el área clínica.

Debido a que la evaluación de la adhesión no es concluyente para determinar el comportamiento de un material, es importante también analizar el tipo de falla que estos adhesivos producen en el momento de la descementación con el fin de determinar su verdadero efecto sobre el esmalte dental. Por tal motivo, el objetivo de este trabajo de investigación fue comparar la fuerza y tipo de falla adhesiva que presentan los sistemas de grabado total (quinta generación) y autograbadores (séptima generación) empleados en ortodoncia, mediante una maquina universal de ensayos y el estéreo-microscopio.

\section{MATERIALES Y MÉTODOS}

Se realizó una investigación experimental In vitro con 20 primeros premolares superiores humanos derechos e izquierdos extraídos por motivos ortodóncicos. Para determinar el tamaño de la muestra se utilizó la fórmula de varianza mancomunada. Los premolares fueron recolectados en diferentes clínicas de la ciudad de Bogotá, un muestreo por conveniencia. Para este estudio se incluyeron premolares que no presentaran alteraciones en el esmalte, caries dental, enfermedad pulpar o periodontal, ni restauraciones en las caras vestibulares. Los 20 premolares se distribuyeron aleatoriamente en dos grupos de 10 dientes cada uno. Al primer grupo se le denominó grabado total (grupo control) y al segundo autograbador (grupo experimental).

Los premolares fueron desinfectados con una solución de hipoclorito de sodio al 0,5\% y posteriormente almacenados en una solución salina isotónica con una gota de merthiolate a temperatura ambiente por un tiempo inferior a dos meses. Para facilitar la manipulación de los especímenes, las raíces se embebieron en un cubo $(3 \times 1 \times 1 \mathrm{~cm})$ de resina acrílica.

Previo a la cementación, la superficie vestibular de los dientes de ambos grupos fue sometida a una profilaxis, se utilizó una pieza de mano de baja velocidad, cepillo, agua destilada y bicarbonato. Posteriormente, los especímenes se lavaron con agua destilada por 30 segundos y se secaron con aire a presión durante 10 segundos.

\section{Protocolos de cementación}

Durante este procedimiento se tuvieron en cuenta los protocolos y recomendaciones del fabricante de los dos sistemas adhesivos evaluados. Este procedimiento fue realizado por uno de los investigadores 
con mayor experiencia clínica. Los componentes y la composición química del cementante y los sistemas adhesivos utilizados se describen en la Tabla 1.

En el grupo control (Grabado total), se acondicionó la cara vestibular de los premolares con ácido fosfórico al 35\% (Scotchbond ${ }^{\mathrm{TM}}$ PhosphoricEtchant, 3M ESPE, St. Paul, Minnesota, USA) por 15 segundos, se lavó con agua destilada por 30 segundos y se secó con aire a presión. En seguida, se aplicó una capa de adhesivo en la superficie vestibular. Luego, con una espátula para resina se tomó una porción del material cementante (Transbond ${ }^{\mathrm{TM}} \mathrm{XT}$, 3M Unitek, St. Paul, Minnesota, USA) y se aplicó en la superficie de la base del bracket estándar (OrthoOrganizers, California, USA), se posicionó en el centro de la corona clínica del diente mediante el uso de un porta bracket y de un posicionador, se ejerció presión y se retiraron los excesos. Por último, se realizó la fotopolimerización: 20 segundos en la zona distal y 20 segundos en la zona mesial del bracket con una lámpara tipo LED (Ortholux ${ }^{\mathrm{TM}}$, 3M ESPE, St. Paul, Minnesota, USA).

En el grupo experimental (Autograbador), se utilizó el sistema blíster (Transbond ${ }^{\circledR}$ Plus SelfEtching Primer) que consiste en tres compartimentos y un aplicador (Figura 1). En el primer compartimiento se encuentra el primer, en el segundo se encuentra el adhesivo y en el último compartimiento (donde se mezclan las sustancias anteriores) un aplicador. Una vez combinados los componentes, mediante el aplicador se colocó una capa del material en la cara vestibular de los premolares frotando el esmalte durante 5 segundos y luego se aireó suavemente. Después de la aplicación del adhesivo, el protocolo de cementación del bracket fue el mismo que se utilizó en el grupo control.

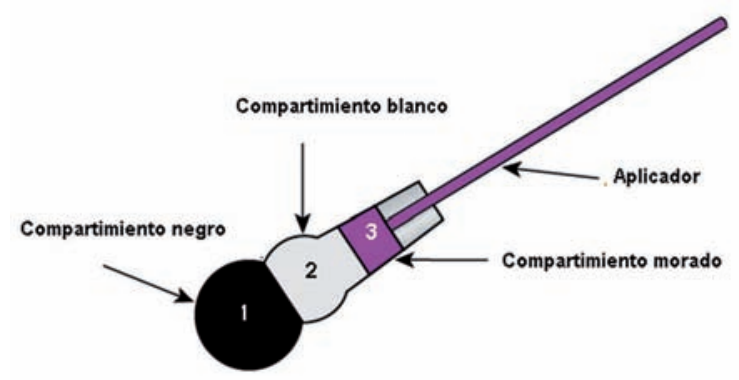

Figura 1. Adhesivo autograbador (Transbond ${ }^{\circledR}$ Plus SelfEtching Primer) en presentación Blister. (1) Primer, (2) Adhesivo y (3) Compartimiento para la mezcla de los dos componentes.

Fuente: Adaptación al español del catálogo de adhesivos de 3M Unitek.

Tabla 1. Descripción del cementante y sus correspondientes sistemas adhesivos.

\begin{tabular}{|c|c|c|c|}
\hline Material & Componentes & Composición Química & Fabricante \\
\hline \multirow{5}{*}{$\begin{array}{c}\text { Transbond }^{\mathrm{TM}} \mathrm{XT} \\
\text { (Cementante) }\end{array}$} & Pasta & Bis-GMA & 3M Unitek (St Paul, MN, USA) \\
\hline & & TEGDMA & \\
\hline & & Silano & \\
\hline & & Sílice de cuarzo & \\
\hline & & Canforoquinona & \\
\hline Transbond ${ }^{\mathrm{TM}} \mathrm{XT}$ & Acondicionador & Ácido fosfórico 35\% & \\
\hline \multirow[t]{2}{*}{ (Adhesivo- Grabado Total) } & Primer-adhesivo & Bis-GMA & 3M Unitek (St Paul, MN, USA) \\
\hline & & $\begin{array}{c}\text { TEGDMA } \\
\text { Fosfonatos metacrilados } \\
\text { Acetona, etanol y agua }\end{array}$ & \\
\hline Transbond $^{\mathrm{TM}}$ & Primer-adhesivo & Metacrilato & \\
\hline $\begin{array}{l}\text { Plus (Adhesivo- } \\
\text { Autograbador) }\end{array}$ & & $\begin{array}{l}\text { Esteres de ácidos } \\
\text { fosfóricos }\end{array}$ & 3M Unitek (St Paul, MN, USA) \\
\hline & & $\begin{array}{l}\text { Canforoquinona } \\
\text { Aminobenzoato }\end{array}$ & \\
\hline
\end{tabular}




\section{Termociclado}

Para esta prueba se utilizó el termociclador, diseñado y fabricado en la Facultad de Odontología de la Pontificia Universidad Javeriana y se siguieron las recomendaciones realizadas en la revisión de la literatura realizada por Amaral y col. en el 2007. ${ }^{18}$ Veinticuatro horas (24h) después de la cementación, los dientes fueron sometidos a 5000 ciclos térmicos. El tiempo de inmersión en cada régimen $\left(5^{\circ}\right.$ y $\left.55^{\circ} \mathrm{C}\right)$ fue de 10 segundos y el tiempo de traslado de 5 a 7 segundos.

\section{Pruebas mecánicas}

Para este procedimiento se utilizó una máquina universal de ensayos (Instron ${ }^{\mathrm{TM}}$ 1011, InstronCorp, Norwood, MA, USA) ubicada en el laboratorio de 3M Colombia. La maquina consta de una cruceta que ejerce una fuerza de corte sobre la interfase esmalte-bracket y unas mordazas neumáticas que sujetan el espécimen. La velocidad de aplicación de la fuerza fue de $1,5 \mathrm{~mm} / \mathrm{min}$, debido a que esta fue la más reportada en la revisión de la literatura realizada por el grupo investigador. El software Blue Hill II (Instron Corp, Norwood MA, USA) registra y procesa la información. Se registró el esfuerzo (MPa) que produjo separación.

\section{Estereomicroscopía}

Una vez descementados los brackets, se procedió a determinar el tipo de falla adhesiva mediante la utilización de un estéreomicroscopio (Stemi 2000C, Carl Zeiss, Göttingen, Germany) ubicado en la Fundación CIEO a una magnificación de 20X. Los dos observadores más experimentados analizaron la superficie vestibular de los premolares y la superficie interna de la base del bracket. El parámetro para realizar este análisis fue la clasificación propuesta por el Medical College of Georgia. ${ }^{19}$ Las cinco posibles interfases de ubicación de la falla se describen a continuación.

\section{- BA: Bracket-Adhesivo \\ (Adhesiva) \\ - AA: Adhesivo-Adhesivo (Cohesiva) \\ - AE: Adhesivo-Esmalte (Adhesiva) \\ - EE: Esmalte-Esmalte (Cohesiva) \\ - ED: Esmalte-Dentina (Adhesiva)}

\section{Análisis estadístico}

Este proceso se realizó mediante los software estadísticos libres R versión 12.2 (2010) y Stat-Plus (2008) para Windows. Después de analizar los datos obtenidos para fuerza adhesiva y el tipo de falla con la prueba de Shapiro Wilk, se determinó que estos datos no provenían de poblaciones con distribución normal. Por lo tanto, se aplicó la prueba de Mann Whitney con una confianza del $95 \%$ para determinar cuál de los dos sistemas adhesivos presentaba la mayor fuerza de adhesión y establecer las diferencias en cuanto al tipo de falla.

\section{RESULTADOS}

Para un fácil entendimiento de los resultados, serán presentados en el mismo orden como se realizaron los procedimientos durante el desarrollo de la investigación.

\section{Fuerza adhesiva}

El adhesivo de grabado total presentó un valor máximo de adhesión de 33,44MPa y un mínimo de 19,08MPa, mientras que el adhesivo autograbador presentó un valor máximo de 22,73MPa y un valor mínimo de 12,09MPa.

$\mathrm{Al}$ comparar los promedios de la fuerza adhesiva entre los dos sistemas se observó una diferencia estadística altamente significativa $(p=0,00008)$, fue superior el valor adhesivo del sistema de grabado total $(28,98 \mathrm{MPa}$ vs.18,67MPa). Los promedios de adhesión obtenidos por los dos adhesivos se observan en la Figura 2.

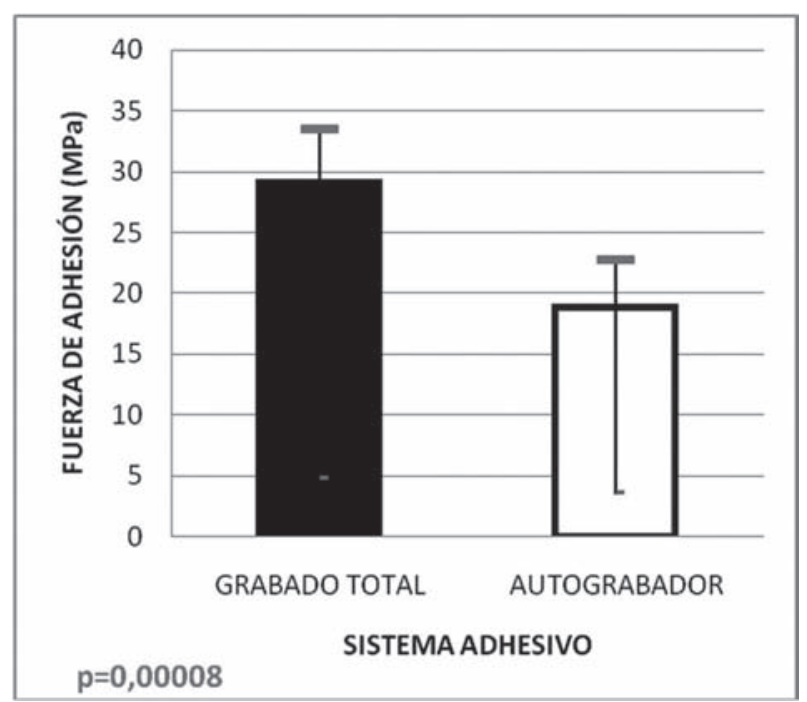

Figura 2. Promedios de fuerza adhesiva obtenidos al utilizar los dos sistemas.

\section{Tipo de falla}

El tipo de falla predominante para ambos sistemas fue en la interfase adhesivo-esmalte (80\% para el sistema de grabado total y $70 \%$ para el autograbador). El resto de fallas se presentaron en la interfase adhesivo-adhesivo ( $20 \%$ y $30 \%$, respectivamente). No se encontró diferencia estadísticamente significativa al comparar el tipo de falla adhesiva presentada por los dos sistemas ( $p>0.05)$. En la Figura 3 se describen los dos tipos de falla observados en ambos grupos. 

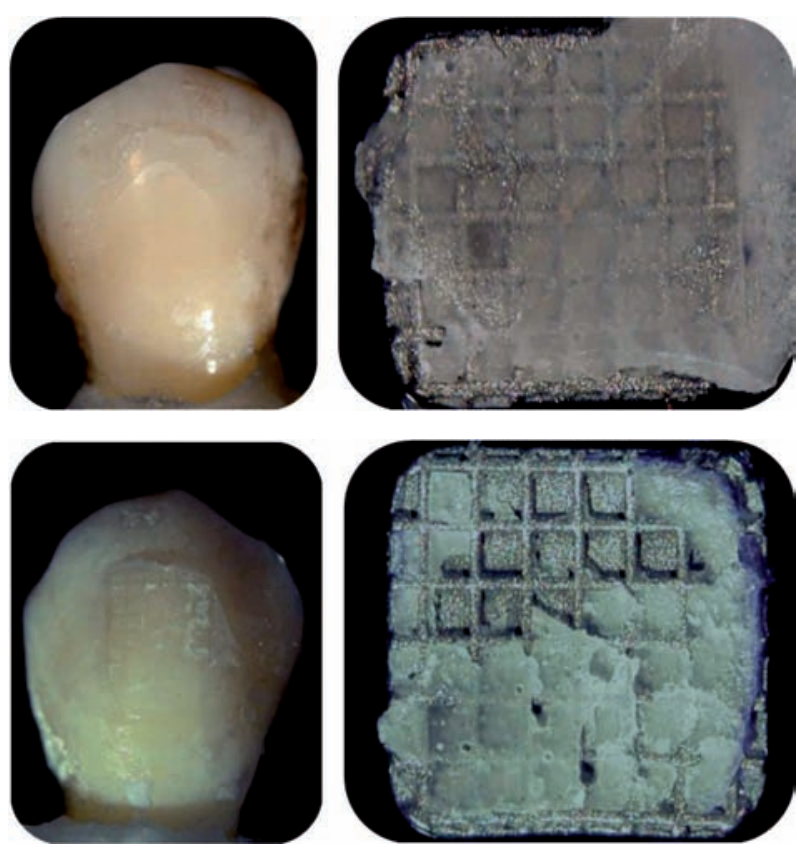

Figura 3. Falla adhesiva en la interfase adhesivo-esmalte (arriba) en el grupo control grabado total. Falla cohesiva en la interfase adhesivo-adhesivo (abajo) en el grupo experimental autograbador.

\section{DISCUSIÓN}

Al comparar los resultados de la prueba realizada para determinar la fuerza adhesiva entre el sistema de grabado total y el autograbador, se observó una diferencia estadísticamente significativa, lo que posiblemente puede estar relacionado con la composición química del material adhesivo y el tipo de acondicionamiento producido por este en el sustrato dentario.

El sistema de grabado total está compuesto de un ácido fuerte, ácido ortofosfórico al $37 \%$, que produce patrones de desmineralización tipo I y II, y genera en el tejido adamantino microporos y microsurcos capilares que miden entre 10 a $25 \mu \mathrm{m}$ de profundidad con una amplitud de 1.5 a $3.5 \mu \mathrm{m}$. Esta topografia es ideal para lograr una mejor retención micromecánica y produce una desmineralización profunda. ${ }^{2}$ Una de las características del esmalte grabado con ácido ortofosfórico es su rugosidad.

Por otro lado, a diferencia del sistema adhesivo de grabado total, el sistema autograbador tiene dentro de su composición un ácido débil, esteres de ácido fosfórico metacrilado y un monómero hidrófilo que según estudios previos producen una retención más superficial en el esmalte debido a la presencia de restos orgánicos en la superficie,,$^{10,14}$ lo cual podría ser un indicador de un grabado ácido insuficiente. Lo anterior llevaría a pensar que la penetrabilidad del agente adhesivo y la humectación que este produce sobre la superficie adamantina están directa- mente relacionadas con los valores de adhesión.

En la literatura científica existen reportes en donde al comparar estos dos sistemas adhesivos se presentan valores de adhesión inferiores a los ob-

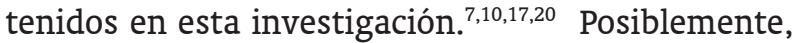
esto puede estar relacionado con la utilización de dientes bovinos y no de dientes humanos. Previamente, Oesterle y colaboradores analizaron el uso de dientes bovinos en estudios de adhesión, concluyeron que las fuerzas observadas eran menores en comparación a las logradas en dientes humanos. ${ }^{21}$ Sin embargo, esto es aún controversial debido a que macroscópicamente como microscópicamente estos presentan características similares. ${ }^{22}$ La utilización de primeros premolares superiores humanos en este estudio fue relevante debido a la uniformidad de sus características morfológicas.

Aunque en este estudio la fuerza adhesiva del sistema autograbador tuvo un valor inferior (18,67MPa), al sistema de grabado total, el valor promedio de fuerza adhesiva en el grupo control $(28,98 \mathrm{MPa})$ estuvo por encima del límite (20MPa) de fuerza adhesiva propuesta por diferentes autores para producir una retención micro-mecánica suficiente, ${ }^{23,24}$ que permite la unión física entre el adhesivo y el esmalte sin lesionar el tejido adamantino en el momento de la remoción de los brackets. Sin embargo, hay que tener en cuenta que por ser este un estudio In vitro, los valores de adhesión suelen ser más altos debido a que se aíslan algunos factores que pueden influir en el fenómeno de adhesión.

La simulación de los cambios térmicos que se producen en la cavidad oral que generan contracción y expansión de los materiales y causan alteraciones entre la interfase diente-adhesivo es un factor importante al realizar estudios In vitro que involucren protocolos adhesivos. En esta investigación se estableció un protocolo de ciclaje térmico lo más cercano posible a lo que ocurriría clínicamente. Es importante tener en cuenta que los 5000 ciclos realizados, según Amaral y colaboradores corresponderían a seis meses de tratamiento; ${ }^{18}$ por lo tanto, los resultados sólo serian aplicables a aproximadamente la tercera parte de la duración frecuente de un tratamiento de ortodoncia correctiva.

Otros autores han utilizado temperaturas similares, ${ }^{25-27}$ sin embargo, han realizado ciclos más cortos y tiempos de inmersión diferentes lo cual limitaría la interpretación del comportamiento en la práctica clínica. Es por eso que para futuros estudios sería interesante determinar la influencia del tiempo de tratamiento sobre la fuerza de adhesión a largo plazo mediante el desarrollo de un proyecto de investigación In vivo que compare diferentes sistemas adhesivos. 
En la prueba mecánica realizada en la maquina universal de ensayos se aplicó una fuerza de corte en dirección ocluso-gingival sobre la base del bracket (interfase esmalte-bracket) debido a que esta es la utilizada con mayor frecuencia en este tipo de estudios lo cual facilita la comparación y el análisis con estudios similares. Klocke y Kahl-Nieke demostraron que los valores de adhesión son estadísticamente diferentes si la fuerza de descementación es aplicada sobre la base del bracket, la ranura de la ligadura o sobre la aleta. ${ }^{28}$

El tipo de falla producido durante el proceso de descementación es relevante al evaluar interfases adhesivas, ya que de esta forma se pueden detectar los factores que pueden causar la falla y así poder determinar si se debe mejorar la resistencia adhesiva (adhesivo-esmalte) o cohesiva (adhesivo-adhesivo) de un material. Con respecto al tipo de falla, los resultados de esta investigación mostraron un predominio en la interfase adhesivo-esmalte para ambos grupos. Este patrón es característico de un tipo de falla adhesiva, lo que clínicamente es favorable ya que es más conservador para el tejido adamantino $y$, a su vez, disminuye el tiempo de trabajo durante la remoción de los excesos del material cementante.

El predominio de falla adhesiva a esmalte en esta investigación difiere de lo reportado por Rikuta y colaboradores. Una posible explicación a esta diferencia se deba a que estos investigadores realizaron la conservación de los especímenes en humedad relativa a diferentes temperaturas durante veinticuatro horas (24h) antes de la prueba mecánica, procedimiento que influyo en la fuerza de adhesión y subsecuentemente en el tipo de falla observada. ${ }^{29}$

A pesar que se utilizó estéreomicroscopia para el análisis de tipo de falla y no se observó ningún tipo de alteración en el esmalte, existen otros métodos de observación tales como la microscopia electrónica de barrido, la cual permite una visualización en tres dimensiones de las superficies, lo que permitiría describir con mayor precisión la ubicación exacta en la cual la falla adhesiva se presenta. Principalmente sería de gran utilidad para determinar la verdadera afectación o no del tejido adamantino.

Al momento de seleccionar un sistema adhesivo se deben tener en cuenta diversos factores tales como el riesgo de contaminación salival,$^{30}$ la disminución del tiempo de trabajo, la capacidad de proporcionar una adhesión suficiente sin causar daño al tejido dentario, la fácil manipulación y el confort tanto para el paciente como para el clínico. De acuerdo a esto, los sistemas adhesivos autograbadores podrían ser una buena opción como material cementante en ortodoncia.
Al tener en cuenta las limitaciones de este estudio In vitro, se concluye que el pre-tratamiento del sustrato dentario y la composición química del adhesivo influye en gran medida con la fuerza de adhesión entre el esmalte y el adhesivo. Así mismo, la fuerza adhesiva obtenida al utilizar sistemas autograbadores en ortodoncia produce una retención micro-mecánica suficiente con un menor riesgo de lesionar el tejido dental.

\section{Agradecimientos}

A la casa comercial 3M Unitek Colombia por facilitar el material cementante, los adhesivos y el dispositivo universal de pruebas (Instron ${ }^{\mathrm{TM}}$ ) utilizado en este estudio. Al comité de investigación de la Fundación Centro de Investigación y Estudios Odontológicos CIEO por su asesoría metodológica y estadística para la realización de este proyecto.

\section{BIBLIOGRAFÍA}

1. Buonocore MG. A simple method of increasing the adhesion of acrylic filling materials to enamel surfaces. J Dent Res 1955; 34: 849-853.

2. Powers JM, Sakaguchi RL. Craig's Restorative Dental Materials. 12 Ed. St Louis: Mosbi Elsevier; 2006. p. 224 - 225.

3. Dorminey J, Dunn W, Taloumis L. Shear bond strength of orthodontic brackets bonded with a modified 1-step etchant -and- primer technique. Am J Orthod Dentofacial Orthop 2003; 124: 410 - 413.

4. Argote C, Rodríguez H, Palau M. Comparación de la fuerza adhesiva de brackets cementados con una resina de fotocurado (Transbond XT con adhesivo MIP) utilizando dos concentraciones de ácido ortofosfórico: al 15\% y al 37\% [Trabajo de Grado]. Bogotá: Universidad Militar Nueva Granada-Fundación CIEO; 2000.

5. Saroğlu I, Aras S, Oztas D. Effect of deproteinization on composite bond strength in hypocalcified amelogenesis imperfecta. Oral Dis 2006; 12: 305 - 358.

6. Espinosa R, Valencia R, Uribe M, Ceja I, Saadia M. Enamel deproteinization and its effect on acid etching: an In vitro study. J Clin Pediatr Dent 2008; 33: 13 - 19.

7. Yamada R, Hayakawa T, Kasai K. Effect of using self-etching primer for bonding orthodontic brackets. Angle Orthod 2002; 72: 558 - 564 .

8. Kaaden C, Powers JM, Friedl KH, Schmalz G. Bond strength of self-etching adhesives to dental hard tissues. Clin Oral Investig 2002; 6: 155 - 160.

9. Hara AT, Amaral CM, Pimenta LA, Sinhoreti MA. Shear bond strength of hydrophilic adhesive systems to enamel. Am J Dent 1999; 12: 181 - 184.

10. Bishara SE, VonWald L, Laffoon JF, Warren JJ. Effect of a self-etch primer/adhesive on the shear bond strength of orthodontic brackets. Am J Orthod Dentofacial Orthop 2001; 119: 621- 624 .

11. Pashley DH, Tay FR. Aggressiveness of contemporary selfetching adhesives Part II: etching effects on unground enamel. Dent Mater 2001; 17: 430 - 444.

12. Murfitt PG, Quick AN, Swain MV, Herbison GP. A randomized clinical trial to investigate bond failure rates using a self-etching primer. Eur J Orthod 2006; 28: 444 - 449. 
13. Kitayama S, Nikaido T, Ikeda M, Foxton RM, Tagami J. Enamel bonding of self-etch and phosphoric acid-etch orthodontic adhesive systems. Dent Mater J 2007; 26: 135 $-143$.

14. Aljubouri YD, Millett DT, Gilmour WH. Laboratory evaluation of a self-etching primer for orthodontic bonding. Eur J Orthod 2003; 25: 411 - 415.

15. Shono Y, Terashita M, Pashley EL, Brewer PD, Pashley DH. Effects of cross-sectional area on resin-enamel tensile bond strength. Dent Mater 1997; 13: 290 - 296.

16. Arnold RW, Combe EC, Warford JH Jr. Bonding of stainless steel brackets to enamel with a new self-etching primer. Am J Orthod Dentofacial Orthop 2002; 122: 274- 276.

17. Dorminey JC, Dunn WJ, Taloumis LJ. Shear bond strength of orthodontic brackets bonded with a modified 1-step etchant-and-primer technique. Am J Orthod Dentofacial Orthop 2003; 124: 410 - 413.

18. Amaral F, Colucci V, Palma-Dibb R, Corona S. Assessment of In vitro methods used to promote adhesive interfase degradation: a critical review. J Esthet Restor Dent 2007; 19: $340-354$.

19. Georgia's Health Sciences University-School of Dentistry. Bond failure site analysis. January 2003.

20. Torii Y, Itou K, Hikasa R, Iwata S, Nishitani Y. Enamel tensile bond strength and morphology of resin-enamel interphase created by acid etching system with or without moisture and self-etching priming system. J Oral Rehabil 2002; 29: 528 - 533.

21. Oesterle LJ, Shellhart WC, Belanger GK. The use of bovine enamel in bonding studies. Am J Orthod Dentofacial Orthop 1998; 114: $514-519$.
22. Posada M, Sánchez C, Gallego G, Vargas A, Restrepo L, López J. Dientes de bovino como sustituto de dientes humanos para su uso en odontología. Revisión de la literatura. Rev CES Odontol 2006; 16: 63 - 68.

23. Keizer S, ten Cate JM, Arends J. Direct bonding of orthodontic brackets. Am J Orthod 1976; 69: 318 - 327.

24. Pickett KL, Sadowsky PL, Jacobson A, Lacefield W. Orthodontic in vivo bond strength: comparison with in vitro results. Angle Orthod 2001; 71:141- 148.

25. Guan G, Takano-Yamamoto T, Miyamoto M, Hattori T, Ishikawa K, Suzuki K. Shear bond strengths of orthodontic plastic brackets. Am J Orthod Dentofacial Orthod 2000; 117: $438-443$.

26. Grubisa HS, Heo G, Raboud D, Glover KE, Major PW. An evaluation and comparison of orthodontic bracket bond strengths achieved with self-etching primer. Am J Orthod Dentofacial Orthop 2004; 126: 213-219.

27. Saito K, Sirirungrojying S, Meguro D, Hayakawa T, Kasai $\mathrm{K}$. Bonding durability of using self-etching primer with 4-META / MMA-TBB resin cement to bond orthodontic brackets. Angle Orthod 2005; 75: 260 - 265.

28. Klocke A, Kahl-Nieke B. Influence of force location in orthodontic shear bond strength testing. Dent Mater 2005; 21:391- 396.

29. Rikuta A, Yoshida T, Tsubota K, Tsuchiya H, Tsujimoto A, Ota M, Miyazaki M. Influence of environmental conditions on orthodontic bracket bonding of self-etching systems. Dent Mater J 2008; 27:654 - 659.

30. Maia SR, Cavalli V, Liporoni PC, do Rego MA. Influence of saliva contamination on the shear bond strength of orthodontic brackets bonded with self-etching adhesive systems. Am J Orthod Dentofacial Orthop 2010; 138: 79 - 83.

\section{Correo electrónico de los autores:}

Ana Lucía Ciceri: anaciceri80@yahoo.es, John Jairo Monroy Parada: johnjairo5050@hotmail.com, Gerardo Ardila Duarte: g.ardila@cieo.edu.co, Antonio Luna Salguero: antonioluna94@gmail.com, Jaime Rodrigo Rivera Barrero: jr.rivera@cieo.edu.co 